\title{
The Development and Validation of a Questionnaire Measuring Digital Eye Strain and Risk Level (DESRIL-27)
}

\section{Zubda Hamid}

Universiti Brunei Darussalam

sharimawati sharbini ( $\square$ shahrimawati.sharbini@ubd.edu.bn )

Universiti Brunei Darussalam

Hanif Abdul Rahman

Universiti Brunei Darussalam

Fazean Irdayati Idris

Universiti Brunei Darussalam

lin naing

Universiti Brunei Darussalam

\section{Research Article}

Keywords: Development, Questionnaire, digital eye strain, musculoskeletal disorders, validation, risk factors, working population

Posted Date: January 18th, 2023

DOI: https://doi.org/10.21203/rs.3.rs-1342108/v2

License: (c) (1) This work is licensed under a Creative Commons Attribution 4.0 International License. Read Full License 


\section{Abstract}

Background: Daily exposure and overuse of digital devices are associated with several health-related issues to human eye, muscles and bones that are collectively termed as Digital Eye Strain and related Musculoskeletal Disorders. These problems have become worse during covid-19 pandemic due to global shift towards working online. This paper introduces a validated research instrument called DESRIL-27 to be used in assessing digital eye strain and related musculoskeletal disorders, with their associated risk factors in the working population.

Methods: DESRIL-27 was developed from thorough review of salient literatures and in consultation with a team of experts to ensure the content validity. This 27 -item tool consists of two scales, namely symptom severity and risk level scales. Reliability and validity analyses were conducted to assess the psychometric properties of DESRIL-27.

Results: Cronbach's alpha was 0.91 for Symptom Severity and 0.88 for Risk Level from the scales in the questionnaire. Principal Component Analysis results ranged from acceptable to very good.

Conclusions:The procedures adopted for the validation and the results of the analysis reveal that DESRIL27 is a reliable and valid research instrument. It can be safely used in future research studies to assess the visual and musculoskeletal problems and their risk factors in digital screen users. The resultant knowledge can help individuals, health practitioners, and related policy makers to prevent and control these problems at workplace.

\section{Background}

The increasing use of digital devices for work and social purposes in response to technological advancement, often for prolonged hours each day, is now normal among individuals of all ages. Research shows that using digital devices (desktops, laptops, tablets, e-readers and cell phones) for more than 4 hours daily, increases the risk of developing a clinical syndrome called Computer Vision Syndrome or CVS $[1,2]$. It has been defined as "a group of eye- and vision-related problems that result from prolonged computer, tablet, e-reader and cell phone use" [3]. CVS is synonymous with the terms Visual Fatigue (VF) and Digital Eye Strain (DES), reflecting the variety of digital devices to be the potential source of these problems [4]. DES encompasses a range of ocular and visual symptoms, which include eyestrain, tired eyes, sore eyes, dry eyes, watery eyes, irritated eyes, blurred vision, and double vision $[2,5]$. Computer use has also been reported to be associated with a range of musculoskeletal disorders (MSD) especially pain, stiffness and numbness in neck, shoulders and back [6]. A systematic review revealed that digital devices, specifically the use of smartphones, may contribute to the occurrence of musculoskeletal changes in the head-neck, shoulder-arm, and handthumb areas [7]. The substantial growth in digital device usage is especially evident during the COVID-19 pandemic, with home-isolation and social distancing leading to a necessitated use of digital devices to work or study online, posing a greater risk of developing digital eye strain-related symptoms as well 
as numerous social, psychological and physical issues. While symptoms are usually transient, the condition can cause significant and frequent discomfort for sufferers, leading to increased disability, reduced occupational productivity and absenteeism from the workplace $[8,9]$.

Digital eye strain has been regarded as one of the biggest occupational hazards of the century [10]. Globally, around 60 million people suffer from DES and an estimated 1 million new cases occur each year [11]. A systematic review of 104 studies on DES between 1980 - 2014 (around the period where computers have become a necessity to daily life) reported the prevalence of DES to range between 64$90 \%$ of the population and has been accounted as the most reported health problem in $70 \%$ of computer users [2]. According to The Occupational Safety and Health Administration of the US Government (OSHA), $90 \%$ of 70 million US workers who use computers for more than three hours per day, experience some form of CVS $[12,13]$. Meanwhile, a study reported that $22 \%$ of computer workers complained of various musculoskeletal disorders such as neck, back and shoulder pains, and carpal tunnel syndrome, attributed mainly due to poor workstation ergonomics and long sitting periods [14].

Many of the current validated questionnaires on DES assess vision related symptoms only and do not include items for musculoskeletal symptoms or risk factors for DES $[15,16]$. Hence, researchers had to use multiple questionnaires in their studies to assess DES and associated risk factors $[17,18]$. In this study, we report the development and validation of a questionnaire called the Digital Eye Strain and Risk Level Questionnaire (DESRIL-27) that incorporates visual and musculoskeletal symptoms of DES and the assessment of risk factors.

\section{Methods}

\section{Study Design}

A questionnaire validation study was conducted in three stages: designing the questionnaire by experts, pretesting questionnaire to assess comprehensibility and finally, psychometric assessment. The study protocol was conducted in accordance to the Declaration of Helsinki and ethical approval was obtained from the PAPRSB Institute of Health Sciences Research Ethics Committee (IHSREC) and University Research Ethics Committee (UREC), Universiti Brunei Darussalam (UBD/PAPRSBIHSREC/2021/11).

\section{Designing the questionnaire by expert committee}

The questionnaire was designed using the "Table of Specification" approach [19-21] to ensure content validity $[22,23]$. The approach started with setting the scope of measurement scale. The first scale (Symptom Severity Scale) measured symptoms related to eye, vision and musculoskeletal system. The second scale (Risk Level Scale) measured risk level regarding work environment (workspace, device or equipment, work hours/breaks/exercises) and ergonomic factors, specifically workstation set-up in digital screen users (Table 1). The areas in each scale were discussed and agreed upon, followed by points in each area. Finally, corresponding items were identified by experts for each point. In addition, we assessed the available items from existing validated questionnaires and adopted relevant items, for example, 
Computer Vision Syndrome Questionnaire (CVS-Q) and Computer Vision Symptom Scale (CVSS17) [15, 16] and recommendations from American Optometric Association and Centers for Disease Control and Prevention $[3,24]$. A total of 36 items were included in the first draft of the questionnaire and the number reduced to 27 items in the final questionnaire.

\section{(Please insert Table 1 here)}

\section{Pretesting the questionnaire}

A comprehensibility assessment (pretesting) of the drafted questionnaire was conducted to check the ease of understanding and acceptability for all components of the questionnaire including introduction, instructions, items and responses [23]. We conducted two sessions of pretests including a total of 14 participants. The participants for both sessions were recruited by purposive sampling and were comprised of administrative staff of Universiti Brunei Darussalam (UBD) from a low educational background, using digital screens regularly and were cooperative to give feedback to the questionnaire. After the first session, the questionnaire was revised according to the feedback given by the participants followed by a second session, which was conducted with the revised version. The questionnaire was revised again according to the feedback from the second session. We removed four items which were identified to have poor comprehensibility with participants and better similar items were present in the questionnaire. The revised questionnaire had 32 items.

\section{Psychometric assessment}

Following pre-testing, we conducted a psychometric assessment to determine whether the items should be revised, omitted or kept unchanged [23]. The revised questionnaire with 32 items was tested among 42 members of administrative staff of UBD from various educational backgrounds, who had been working with digital screens for at least one year and were recruited by purposive sampling. The purpose of sampling here, was to recruit a range of participants from those who had minimal use of digital screen to those who had maximal use, almost all the time. The members who participated in pretests were not included in this study. A researcher was present to address any queries and clarifications by the participants who answered the paper-based questionnaire. With the results of psychometric analysis, the questionnaire was revised, and the final revision was obtained which had 27 items; 16 items in Symptom Severity Scale and 11 items in Risk Level Scale (Table 1).

\section{Scoring of the questionnaire}

Symptom Severity Scale has two components, frequency and intensity. There are four responses for frequency of symptom: never, sometimes, often and almost always, and are given scores 0, 1, 2 and 3 respectively. Intensity scale has four responses: mild, moderate, intense and very intense, and are given scores 1, 2, 3 and 4 respectively. Each item score was calculated by multiplying frequency and intensity scores, having values from 0 to 12 . Therefore, the Symptom Severity Scale ranges from 0 to 192 for the 16 item-scale. Higher score reflects higher symptom severity. 
For Risk Level Scale, there are 11 items with 4 responses. Three items have the following responses: very comfortable, comfortable, slightly uncomfortable, and very uncomfortable, and are given a score of 0 to 3 respectively. Remaining 8 items have the following responses: more than adequate, adequate, slightly inadequate, and very inadequate and are given a score of 0 to 3 respectively. Therefore, the Risk Level Scale ranges from 0 to 33 for the 11 item-scale. Higher score reflects higher risk level.

\section{Statistical Analysis}

All analysis was conducted using R (ver. 4.1.1) and RStudio for Mac (ver. 4.0.2). The package "psych" was used to assess psychometric properties of the questionnaire [25]. Quantitative variables were presented with means and standard deviations or median and IQR (Interquartile range) as appropriate. Qualitative variables were presented with frequency distribution and percentages. Analyses on psychometric properties included internal consistency reliability analysis for reliability, principal component analysis for construct validity and item and scale level descriptive statistics to assess the sample distribution adequacy.

For internal consistency reliability, Cronbach alpha value of $\geq 0.70$ is considered as having a good reliability and corrected item-total correlations were used to assess each item property [26]. Construct validity assesses the extent to which a scale measures what it intends to measure [27], with its two components, convergence and divergence validity. Principal component analysis (PCA) was used to assess whether items have convergent and discriminant validity [28]. In this study, if an item was loaded (factor loading $\geq 0.40$ ) on a component that appeared to be the scale intended, it was considered a good convergent validity. On the other hand, if the item was not loaded (factor loading $<0.20$ ) on other components, it was considered a good discriminant validity.

Each scale was described with mean and standard deviation, minimum and maximum score, and floor and ceiling percentages. Floor percentage was calculated as the percentage of respondents at the lowest scale point (score zero for Symptom Severity Scale and zero for Risk Level Scale). Ceiling percentage was calculated as the percentage of respondents at the highest scale point (score 192 for Symptom Severity Scale and 33 for Risk Level Scale). These statistics assess the adequacy of purposive sampling that aims to have a good range of sample in the validation study.

\section{Results}

A total of 42 respondents participated in the psychometric assessment. The median age of the participants was 35.5 years, and all were full-time employees working in the administration offices of UBD. Majority of the respondents was females (64.3\%), education below bachelor degree (88.1\%), income less than Brunei Dollars (BND) 2000 (88.1\%), using spectacles (62.0\%), had no regular eye checkup $(81.0 \%)$ and used digital screens for more than 5 hours per day (66.7\%). Detailed description of the respondents is presented in Table 2.

(Please insert Table 2 here) 


\section{Psychometric properties of the questionnaire}

Initially, 32 items were analyzed for psychometric properties. There were three poorly performed items, with corrected item-total correlation and poor factor loadings ( $<0.40$ in both) on the respective component in Principal Component Analysis. Items' review showed that there were similar items available. Therefore, these three items were dropped from the questionnaire, as it did not compromise the content validity.

As the Cronbach's alpha was still high, 0.91 for Symptom Severity Scale and 0.88 for Risk Level Scale, we identified similar items in the questionnaire and dropped two items without compromising the content validity. Finally, with the 27 -item questionnaire, it satisfied a good reliability and construct validity with some minor or acceptable weakness. There were four items S2, S5, S8 and RL1, which we considered to have marginally acceptable corrected item-total correlation whereas their factor loading was good in respective component. The eigen value extracted by Component 1 was 7.50 units and by Component 2 was 5.94 units. Variance extracted by Component 1 was $28 \%$ and by Component 2 was $22 \%$, giving a total of $50 \%$ of variance extracted by the two components. We present details of psychometric statistics of the DESRIL-27 in Table 3.

Table 4 shows descriptive statistics of Symptom Severity and Risk Level Scales. It revealed that for the Symptom Severity Scale, the lowest score was 1 and the highest score was 97, while the scale ranges from 0 to 192. The sample taken occupied well in the lower half of the scale but not adequately occupied in the upper half of the scale. With regard to Risk Level Scale, the lowest score was 1 and the highest score was 28 , while the scale ranges from 0 to 33 . Though it did not reach the lowest and highest of the scale, the sample taken spread or occupied well in the scale range. However, the psychometric analysis gave a good outcome in this study.

\section{Discussion}

In this validation study, the questionnaire was drafted ensuring content validity using relevant experts, literature review, and proper process, that is, Table of Specification. Secondly, the draft was tested for comprehensibility with two groups of target population. In this way, jargon, technical terms, ambiguous statement, double barrel items, etc. were removed to minimise measurement errors. Then the improved version was assessed using statistical analyses for reliability and validity. This validation methodology guides toward a certain degree of valid questionnaire.

The instrument has two scales: the severity of symptoms and risk level for DES and MSD. For the Internal Consistency Reliability, the Cronbach's Alpha coefficient for symptom severity scale was 0.91 and for risk level scale was 0.88 , while the Principal Component Analysis proved the discriminant and convergent validity of the questionnaire. We demonstrated that DESRIL-27 measured two scales with excellent reliability and validity on a sample of administrative staff of UBD.

While reviewing the literature on effects of digital screen use on human eye and musculoskeletal system, it was found that there is a gap in the knowledge to understand the symptoms and risk factors due to 
lack of validated questionnaires. Several studies used instruments which did not follow the standard patterns of questionnaire validation and due to this reason, there is discrepancy among the results, with prevalence of DES to be $19.6 \%$ in one study, and more than $80 \%$ in another study $[29,30]$.

When we compare our instrument to other validated questionnaires, it is found that the reliability of DESRIL-27 is superior to CVS-Q and is equivalent to CVSS17. For our instrument, the reliability coefficient (Cronbach's alpha) for symptom severity of DES is 0.91 whereas for CVS-Q and CVSS17, it is 0.78 and 0.92 respectively. Regarding ICC (Intraclass correlation) which is done for test-retest reliability [31], the CVS-Q has a value of 0.80 and CVSS17 has a value of 0.85 . It shows that the ICC values of both these instruments are also comparable to our reliability coefficient $[15,16]$.

This newly developed and validated questionnaire DESRIL-27 is unique in the sense that, in addition to eye problems, it includes items to assess the effects of musculoskeletal symptoms associated with the use of digital screens, which other questionnaires such as CVS-Q and CVSS-17 lack in $[15,16]$. Hence, this questionnaire can be used as a guide for employers to create awareness on the importance of fulfilling the ergonomic requirements while at work with digital screens.

The methodology used for the questionnaire validation is considered to be the main strength of the study. All steps followed in this study were according to the standard practice of validation of health questionnaires [27]. Both pretests and the psychometric assessment were conducted in the presence of the researchers. The questionnaire was paper based, easy to understand, with a 12-minute completion time on average.

A limitation of the study is that the diagnosis of DES is through self-reporting where reporting bias could arise. There could also be some variation in different culture and nature of workplace which could introduce measurement errors. Therefore, this questionnaire should be further evaluated in other populations and also in different work settings in future studies.

With COVID-19 pandemic continuing its presence, working or schooling from home and communication using digital technologies will inevitably be part of the new norm, where many individuals may suffer from the physical effects of overuse of digital devices. By gaining information on the symptom severity and risk levels, individuals may get away from prolonged use of digital devices. This may guide them to be more empowered in management of their health by ensuring visual and musculoskeletal health awareness. In addition, employers can explore strategies to manage DES and MSD effectively in the workplace such as adopting proper ergonomic measures, incorporating regular screen breaks and practicing the 20-20-20 rule in the workplace [32]. Good health of workers is not only beneficial for themselves but also critical for the growth of the organizations as well as the society as a whole.

Future studies on impact of digital devices and health can include assessment of ergonomic and environmental factors. Comparative studies can also be done among different occupations in the same country and among same occupations in different countries. This will not only help determine the magnitude of DES and MSD but also the associated risk factors in other parts of the world. 
The researchers have prepared a manual and a coding guideline for the scoring items to assist the researchers in conducting their future studies using DESRIL-27. All this material along with the questionnaire are available on our website (https://sites.google.com/view/thedesril-27project/).

\section{Conclusion}

This study has successfully shown that DESRIL-27 is a reliable and valid instrument for assessment of DES and MSD symptom prevalence and risk factors. DESRIL-27 provides opportunities for other researchers to conduct prevalence studies on DES and MSD among individuals from various professions working with digital screens. This in turn may lead to further research opportunities to determine the causative factors for DES and MSD due to digital screen use. By doing so, this will enable stakeholders to manage the visual and musculoskeletal health of employees by implementing necessary modifications in the work environment.

\section{Abbreviations}

CVS: Computer Vision Syndrome

VF: Visual Fatigue

DES: Digital Eye Strain

MSD: Musculoskeletal Disorders

UBD: Universiti Brunei Darussalam

IQR: Interquartile range

PCA: Principal Component Analysis

BND: Brunei Dollar

ICC: Intraclass Correlation

\section{Declarations}

\section{Ethics approval and consent to participate}

The study protocol and methods were conducted in accordance to the Declaration of Helsinki and ethical approval was obtained from the PAPRSB Institute of Health Sciences Research Ethics Committee (IHSREC) and University Research Ethics Committee (UREC), Universiti Brunei Darussalam (UBD/PAPRSBIHSREC/2021/11). The study received full approval from the PAPRSB Institute of Health Sciences Research Ethics Committee (IHSREC) and University Research Ethics Committee (UREC), Universiti Brunei Darussalam (UBD/PAPRSBIHSREC/2021/11). A clear and detailed participant 
information sheet was given to potential participants after which written informed consent was signed by all participants prior to study being started.

\section{Consent for publication}

Not applicable

\section{Availability of data and materials}

The datasets generated and/or analysed during the current study are not publicly available due to privacy policy of Ethics Committee of Universiti Brunei Darussalam. It allows only to publish findings without sharing data to any parties. Data can be accessed by contacting Sharimawati Sharbini by email shahrimawati.sharbini@ubd.edu.bn

The DESRIL-27, manual and scoring strategy is available at https://sites.google.com/view/thedesril27project/

\section{Competing interests}

The authors declare that they have no competing interests.

\section{Funding}

The authors declare that no funding is involved in this study project.

\section{Authors' contribution}

ZH: Concept, study design, data collection, data analysis, paper writing

SS: Concept, study design, data collection, paper writing

HAR: Concept, study design, data collection, paper writing

FI: Concept, paper writing and editing

LN: Concept, study design, data analysis, paper writing

All authors read and approved the final manuscript.

\section{Corresponding author}

Correspondence to Sharimawati Sharbini (shahrimawati.sharbini@ubd.edu.bn)

\section{Acknowledgements}

We acknowledge the administration staff of Universiti Brunei Darussalam for their technical support and participation in the study. We are grateful to Dr Liyana Abdulla (Orthoptist - RIPAS Hospital) and Dr NBP 
Balalla (Occupational Health Specialist - JPMC) for their guidance during questionnaire development and validation. We also acknowledge the services provided by Haji Hazilan Ramli (Head OSHE - UBD) for data collection during Pilot study.

\section{Authors' Information}

Affiliations

PAPRSB Institute of Health Sciences, Universiti Brunei Darussalam, Tungku Link Road, Gadong BE 1410, Brunei Darussalam

Zubda Hamid, Sharimawati Sharbini, Hanif Abdul Rahman, Fazean Irdayati Idris \& Lin Naing

\section{References}

1. Khola Noreen Z, Batool, Tehreem Fatima TZ. Prevalence of Computer Vision Syndrome and Its Associated Risk Factors among Under Graduate Medical Students of Urban Karachi. Pakistan J Ophthalmol. 2016;32:140-6.

2. Akinbinu T, Mashalla YJ. Impact of computer technology on health: Computer Vision Syndrome (CVS). Med Pract Rev. 2014;5:20-30.

3. American Optometric Association. Computer vision syndrome. https://www.aoa.org/healthyeyes/eye-and-vision-conditions/computer-vision-syndrome?sso=y. Accessed 27 Mar 2021.

4. Rosenfield M. Computer vision syndrome: A review of ocular causes and potential treatments. Ophthalmic Physiol Opt. 2011;31:502-15.

5. Nichols JJ, Ziegler C, Mitchell GL, Nichols KK. Self-reported dry eye disease across refractive modalities. Investig Ophthalmol Vis Sci. 2005;46:1911-4.

6. Lanhers C, Pereira B, Garde G, Maublant C, Dutheil F, Coudeyre E. Evaluation of "I-Preventive": a digital preventive tool for musculoskeletal disorders in computer workers-a pilot cluster randomised trial. BMJ Open. 2016;6:e011304.

7. Eitivipart AC, Viriyarojanakul S, Redhead L. Musculoskeletal disorder and pain associated with smartphone use: A systematic review of biomechanical evidence. Hong Kong Physiother J. 2018;38:77-90.

8. Gouveia N, Rodrigues A, Eusébio M, Ramiro S, Machado P, Canhão H, et al. Prevalence and social burden of active chronic low back pain in the adult Portuguese population: results from a national survey. Rheumatol Int. 2016;36:183-97.

9. Smith TST, Frick KD, Holden BA, Fricke TR, Naidoo KS. Potential lost productivity resulting from the global burden of uncorrected refractive error. Bull World Health Organ. 2009;87:431-7.

10. Charpe NA, Kaushik V. Computer Vision Syndrome (CVS): Recognition and Control in Software Professionals. J Hum Ecol. 2009;28:67-9. doi:10.1080/09709274.2009.11906219. 
11. Sen A, Richardson Musc S. A study of computer-related upper limb discomfort and computer vision syndrome. J Hum Ergol. 2007;36:45-50.

12. Bali J, Navin N, Thakur BR. Computer vision syndrome: A study of the knowledge, attitudes and practices in. Indian Ophthalmologists. 2007;55:289-93.

13. Blehm C, Vishnu S, Khattak A, Mitra S, Yee RW. Computer vision syndrome: A review. Surv Ophthalmol. 2005;50:253-62.

14. Hales TR, Sauter SL, Peterson MR, Fine LJ, Putz-Anderson V, Schleifer LR, et al. Musculoskeletal disorders among visual display terminal users in a telecommunications company. Ergonomics. 1994;37:1603-21.

15. González-Pérez M, Susi R, Antona B, Barrio A, González E. The Computer-Vision Symptom Scale (CVSS17): Development and initial validation. Investig Ophthalmol Vis Sci. 2014;55:4504-11.

16. Seguí MDM, Cabrero-García J, Crespo A, Verdú J, Ronda E. A reliable and valid questionnaire was developed to measure computer vision syndrome at the workplace. J Clin Epidemiol. 2015;68:66273.

17. Cantó-Sancho N, Sánchez-Brau M, Ivorra-Soler B, Seguí-Crespo M. Computer vision syndrome prevalence according to individual and video display terminal exposure characteristics in Spanish university students. Int J Clin Pract. 2021;75. doi:10.1111/ijcp.13681.

18. Zayed HAM, Saied SM, Younis EA, Atlam SA. Digital eye strain: prevalence and associated factors among information technology professionals, Egypt. Environ Sci Pollut Res Int. 2021;28:25187-95.

19. Turocy PS. Survey Research in Athletic Training: The Scientific Method of Development and Implementation. J Athl Train. 2002;37(4 Suppl):174-9.

20. Goddard RD, Villanova P. Designing surveys and questionnaires for research. In: The psychology research handbook: A guide for graduate students and research assistants. 2006. p. 114-25.

21. Murray P. Fundamental issues in questionnaire design. Accid Emerg Nurs. 1999;7:148-53.

22. Rajabi-Vardanjani H, Habibi E, Pourabdian S, Dehghan H, Maracy MR. Designing and validation a visual fatigue questionnaire for video display terminals operators. Int J Prev Med. 2014;5:841-8.

23. Tsang S, Royse CF, Terkawi AS. Guidelines for developing, translating, and validating a questionnaire in perioperative and pain medicine. Saudi J Anaesth. 2017;11(Suppl 1):80-9.

24. CDC: Vision Health Initiative (VHI). 2021. https://www.cdc.gov/visionhealth/index.htm. Accessed 4 May 2021.

25. Revelle W. An overview of the psych package. Dep Psychol Northwest Univ. 2011;3:1-25.

26. Cronbach LJ, Shavelson RJ. My Current Thoughts on Coefficient Alpha and Successor Procedures. Educ Psychol Meas. 2004;64:391-418. doi:10.1177/0013164404266386.

27. Streiner DL, Norman GR, Cairney J. Health measurement scales: a practical guide to their development and use. USA: Oxford University Press; 2015.

28. Litwin M. How to Measure Survey Reliability and Validity. Thousand Oaks: SAGE Publications, Inc; 1995. doi:10.4135/9781483348957. 
29. Tamez González S, Ortiz-Hernández L, Martínez-Alcántara S, Méndez-Ramírez I. Risks and health problems caused by the use of video terminals. Salud Publica Mex. 2003;45:171-80.

30. Ye Z, Honda S, Abe Y, Kusano Y, Takamura N, Imamura Y, et al. Influence of work duration or physical symptoms on mental health among Japanese visual display terminal users. Ind Health. 2007;45:328-33.

31. Koo TK, Li MY. A guideline of selecting and reporting intraclass correlation coefficients for reliability research. J Chiropr Med. 2016;15:155-63.

32. AOA: 20-20-20-Rule to prevent Digital Eye Strain. 2017. https://www.aoa.org/AOA/Images/Patients/EyeConditions/20-20-20-rule.pdf. Accessed 22 Dec 2021.

33. Bali J, Navin N, Thakur BR. Computer vision syndrome: A study of the knowledge, attitudes and practices in Indian Ophthalmologists. Indian J Ophthalmol. 2007;55:288-94.

34. Rahman ZA, Sanip S. Computer User: Demographic and Computer Related Factors that Predispose User to Get Computer Vision Syndrome. Int J Business Humanit Technol. 2011;1:84-91.

35. Kuorinka I, Jonsson B, Kilbom A, Vinterberg H, Biering-Sørensen F, Andersson G, et al. Standardised Nordic questionnaires for the analysis of musculoskeletal symptoms. Appl Ergon. 1987;18:233-7.

36. Sheppard AL, Wolffsohn JS. Digital eye strain: Prevalence, measurement and amelioration. BMJ Open Ophthalmol. 2018;3.

37. Moodley M, Ismail F, Kriel A. Work-related musculoskeletal disorders amongst undergraduate nursing students at the University of Johannesburg. Heal SA Gesondheid. 2020;25:1-9. doi:10.4102/hsag.v25i0.1460.

38. Janwantanakul P, Pensri P, Jiamjarasrangsri V, Sinsongsook T. Prevalence of self-reported musculoskeletal symptoms among office workers. Occup Med (Chic III). 2008;58:436-8.

39. CCOHS: Office Ergonomics; OSH Answers Fact Sheets. 2021. https://www.ccohs.ca/oshanswers/ergonomics/office/. Accessed 29 Oct 2021.

40. Occupational Safety and Health Administration (OSHA). Ergonomics. https://www.osha.gov/ergonomics. Accessed 29 Oct 2021.

\section{Tables}

\section{Table 1. Content validity of DESRIL-27 with Table of Specification}


The first measure/scale: Eye and musculoskeletal symptom severity in digital screen users (16 items)

Areas

Points

Items

In the past 12 months, do you feel the following symptoms during or after digital screen use (Frequency and Intensity)

Symptoms related

to eyes $[3,33,34]$
1. Pain

(20)

2. Irritation
S1. Burning in eyes

S6. Pain in eyes

S13. Headache

S2. Itching in eyes

S4. Watering of eyes

S3. Feeling of something in eyes

3. Blinking

S5. Excessive blinking

Symptoms related to vision $[3,33,34]$

Symptoms related to Musculoskeletal system $[11,35]$
4. Eyesight related

S8. Double vision

S7. Blurred vision

S12. Feeling that eyesight is getting weak

S9. Difficulty focusing

S11. Coloured circles of light around objects

5. Light sensitivity

S10. Increased sensitivity to light

6. Motor symptoms and sensory symptoms related to neck

7. Motor symptoms and sensory symptoms related to shoulders

8. Motor symptoms and sensory symptoms related to back
S14. Pain, Stiffness, Numbness or Tingling sensation in neck

S15. Pain, Stiffness, Numbness or Tingling sensation in shoulders

S16. Pain, Stiffness, Numbness or Tingling sensation in back

The second measure/scale: Risk level regarding work environment and ergonomic factors in digital screen users (11 items)

In the past 12 months, how do you feel about following factors regarding your work environment

Workspace

environment $[11,35]$

Device or equipment

$[3,16,36]$
1. Temperature

RL1. Temperature of the workplace

2. Position

RL4. Distance from computer screen 
3. Display properties RL6. Size of the screen

RL8. Text size

RL7. Quality of display

4. Protective measures RL9. Use of glare reduction filter cover

Work hours / breaks

5. Work hours

RL2. Work hours

$/$ exercises $[37,38]$

6. Breaks

RL10. Short breaks during computer work

7. Exercise

RL11. Stretching exercises during work

Workstation set up and ergonomics [39,

8. Ergonomics

RL3. Position of computer, table height, 40] adjustable chair

Table 2. Descriptive Statistics of 42 respondents 


\begin{tabular}{|c|c|c|}
\hline Variables & $n(\%)$ & Median (IQR) \\
\hline Age (in years) & - & $35.5(15)^{\mathrm{a}}$ \\
\hline \multicolumn{3}{|l|}{ Gender } \\
\hline Male & $15(35.7)$ & \\
\hline Female & $27(64.3)$ & \\
\hline \multicolumn{3}{|l|}{ Ethnicity/Race } \\
\hline Malay & $38(90.5)$ & \\
\hline Chinese & $1(2.4)$ & \\
\hline Others & $3(7.1)$ & \\
\hline \multicolumn{3}{|l|}{ Educational level } \\
\hline Below Bachelor degree & $37(88.1)$ & \\
\hline Bachelor degree (from college or university) & $3(7.1)$ & \\
\hline Higher than Bachelor degree & $2(4.8)$ & \\
\hline \multicolumn{3}{|l|}{ Type of employment } \\
\hline Full time & $42(100)$ & \\
\hline Part time & - & \\
\hline \multicolumn{3}{|l|}{ Monthly income in BND } \\
\hline Less than 2000 & $37(88.1)$ & \\
\hline 2000 to 4000 & $3(7.1)$ & \\
\hline Above 4000 & $2(4.8)$ & \\
\hline \multicolumn{3}{|l|}{ Duration of current employment } \\
\hline 1 to 3 years & $10(23.8)$ & \\
\hline More than 3 years & $32(76.2)$ & \\
\hline \multicolumn{3}{|l|}{ Use of glasses or contact lenses to improve eyesight } \\
\hline Yes & $26(62.0)$ & \\
\hline No & $16(38.1)$ & \\
\hline \multicolumn{3}{|l|}{ Regular eyes checkup (minimum once every year) } \\
\hline Yes & $8(19.0)$ & \\
\hline No & $34(81.0)$ & \\
\hline
\end{tabular}

Page 15/18 


\begin{tabular}{|ll}
\hline Estimated total time spent viewing digital screen per day \\
\hline Less than 3 hours & $4(9.5)$ \\
\hline 3 to 5 hours & $10(23.8)$ \\
\hline More than 5 hours & $28(66.7)$
\end{tabular}

IQR = Interquartile range $\quad \mathbf{a}=$ The distribution is skewed to the right

Table 3: Psychometric properties (Internal consistency reliability and Construct validity) of DESRIL-27 


\begin{tabular}{|c|c|c|c|c|c|c|}
\hline \multirow[t]{2}{*}{ Construct } & \multirow[t]{2}{*}{ Items } & \multirow[t]{2}{*}{ Mean (SD) } & \multicolumn{2}{|c|}{ Construct Validitya } & \multicolumn{2}{|c|}{ IC Reliabilityb } \\
\hline & & & Comp. 1 & Comp. 2 & ITC & Alpha $^{d}$ \\
\hline \multirow{16}{*}{$\begin{array}{l}\text { Symptom } \\
\text { Severity Scale }\end{array}$} & $\mathrm{S} 1$ & $0.6(0.96)$ & 0.63 & - & 0.57 & \multirow[t]{16}{*}{0.91} \\
\hline & $\mathrm{S} 2$ & $1.3(1.40)$ & 0.40 & - & $0.35^{\mathrm{e}}$ & \\
\hline & S3 & $0.7(0.94)$ & 0.68 & - & 0.62 & \\
\hline & S4 & $1.3(1.81)$ & 0.77 & - & 0.74 & \\
\hline & S5 & $0.5(0.92)$ & 0.41 & - & $0.37^{e}$ & \\
\hline & S6 & $0.6(1.71)$ & 0.75 & - & 0.71 & \\
\hline & S7 & $1.0(1.91)$ & 0.82 & - & 0.77 & \\
\hline & S8 & $0.4(1.09)$ & 0.40 & - & $0.34^{\mathrm{e}}$ & \\
\hline & S9 & $1.0(1.37)$ & 0.67 & - & 0.65 & \\
\hline & S10 & $1.8(2.56)$ & 0.89 & - & 0.84 & \\
\hline & $\mathrm{S} 11$ & $0.4(0.62)$ & 0.53 & - & 0.49 & \\
\hline & $\mathrm{S} 12$ & $0.7(1.17)$ & 0.66 & - & 0.60 & \\
\hline & S13 & $1.9(2.73)$ & 0.76 & - & 0.69 & \\
\hline & S14 & $1.4(1.91)$ & 0.72 & - & 0.68 & \\
\hline & S15 & $1.0(1.44)$ & 0.68 & - & 0.63 & \\
\hline & S16 & $1.3(1.74)$ & 0.78 & - & 0.72 & \\
\hline \multirow[t]{10}{*}{ Risk Level Scale } & RL1 & $2.4(0.77)$ & - & 0.43 & $0.38^{e}$ & \multirow[t]{10}{*}{0.88} \\
\hline & RL2 & $2.3(0.79)$ & - & 0.64 & 0.57 & \\
\hline & RL3 & $2.3(0.87)$ & - & 0.54 & 0.48 & \\
\hline & RL4 & $1.9(0.74)$ & - & 0.85 & 0.78 & \\
\hline & RL5 & $2.1(0.96)$ & - & 0.80 & 0.74 & \\
\hline & RL6 & $1.8(0.72)$ & - & 0.71 & 0.61 & \\
\hline & RL7 & $1.8(0.77)$ & - & 0.84 & 0.78 & \\
\hline & RL8 & $1.7(0.80)$ & - & 0.73 & 0.65 & \\
\hline & RL9 & $3.2(1.20)$ & - & 0.60 & 0.51 & \\
\hline & RL10 & $2.1(0.78)$ & - & 0.75 & 0.70 & \\
\hline
\end{tabular}



RL11
$2.0(0.78)$
0.54
0.47

a Data extraction using Principal Component Analysis and Varimax rotation; The factor loading less than

0.40 is suppressed for presentation

b Internal consistency reliability

${ }^{\mathbf{c}}$ ITC = Corrected item total correlation dAlpha = Cronbach's alpha

${ }^{\mathbf{e}}$ Marginally acceptable items

Table 4: Descriptive Statistics of Symptom Severity and Risk Level Scales ( $n=42)$

\begin{tabular}{|llll|}
\hline Construct (Scale) & Mean (SD) & Min., Max. score & Floor\%, Ceiling\% \\
\hline Symptom severity (0 to 192) & $16.0(17.10)$ & 1,97 & 0,0 \\
\hline Risk level (0 to 33) & $12.7(6.27)$ & 1,28 & 0,0 \\
\hline
\end{tabular}

$\mathrm{SD}=$ Standard deviation; Min = Minimum; Max = Maximum

\section{Supplementary Files}

This is a list of supplementary files associated with this preprint. Click to download.

- DESRIL27Manual1d1.docx 